\title{
Laryngeal Ulceration in Behçet's Disease: The Role of Centres of Excellence in the UK - Case Report
}

\author{
Randa Ghazal Asswad ${ }^{1}$, Anna Harrison ${ }^{2}$, Paul Hans ${ }^{2}$ \\ ${ }^{1}$ University of Liverpool Medical School, ${ }^{2}$ ENT Department, Blackpool Victoria Teaching Hospital
}

\section{INTRODUCTION:}

- Behçet's Disease (BD) is a rare complex multisystem inflammatory condition characterized by a triad of symptoms: aphthous ulcers of the mouth and genitalia and uveitis. ${ }^{1}$

- $\quad$ BD is very rare in the UK with a prevalence of 0.64 in every 100,000 inhabitants. ${ }^{2}$

- The literature details few reports of laryngeal ulceration in BD. These cases highlight the potential for significant oedema of the area and the subsequent risk of airway obstruction which is life-threatening. ${ }^{3}$

\section{CASE REPORT:}

- We present the case of a 31-year old Caucasian man who presented to the ENT department on several occasions with severe odynophagia and stridor due to supraglottic oedema with a past history of recurrent mucosal ulcerations of the mouth and larynx for 11 years.

- Examination revealed oral ulcerative lesions (Fig. 1a, 1b) with ulcerative lesions on the epiglottis and the left aryepiglottic fold (Fig. 2a, 2b) and another on the rectal mucosa. Biopsies showed non-specific inflammatory infiltrates with neutrophil predominance. Papular skin lesions and pathergy following blood tests were also reported.

- Infection, malignancy and an auto-immune work-up were all negative. A diagnosis of BD was entertained and the patient referred to The Behçet's Syndrome Centre of Excellence in Liverpool, UK despite not meeting the classification criteria for BD.

- In time he developed genital ulcers and recurrent DVTs despite appropriate anticoagulation, fulfilling the ICBD criteria 11 years after first presentation.

- The treatment plan included immunosuppression with azathioprine, colchicine and low dose cortisone therapy to minimize the recurrence of ulcerations and the patient responded well.

TABLE I

International Study Group for Behçet's Disease (ISGBD) Criteria ${ }^{4}$.

Recurrent oral ulcers

Plus, any two of the following:

Recurrent genital ulcers

Eye lesions

Skin lesions

Positive pathergy test

TABLE II

International Criteria for Behçet's Disease (ICBD) ${ }^{5}$.

\begin{tabular}{lc}
\hline Symptom & Points \\
\hline Oral ulcers & 2 \\
\hline Genital ulcers & 2 \\
\hline Ocular manifestations & 2 \\
\hline Positive skin pathergy test & 1 \\
\hline Skin lesions & 1 \\
\hline Neurological manifestations & 1 \\
\hline Vascular manifestations & 1 \\
\hline
\end{tabular}

A score $\geq 4$ points is needed for a diagnosis of $B D$.

\section{年}

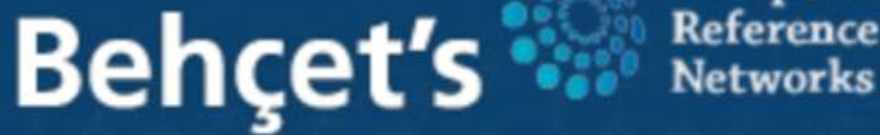

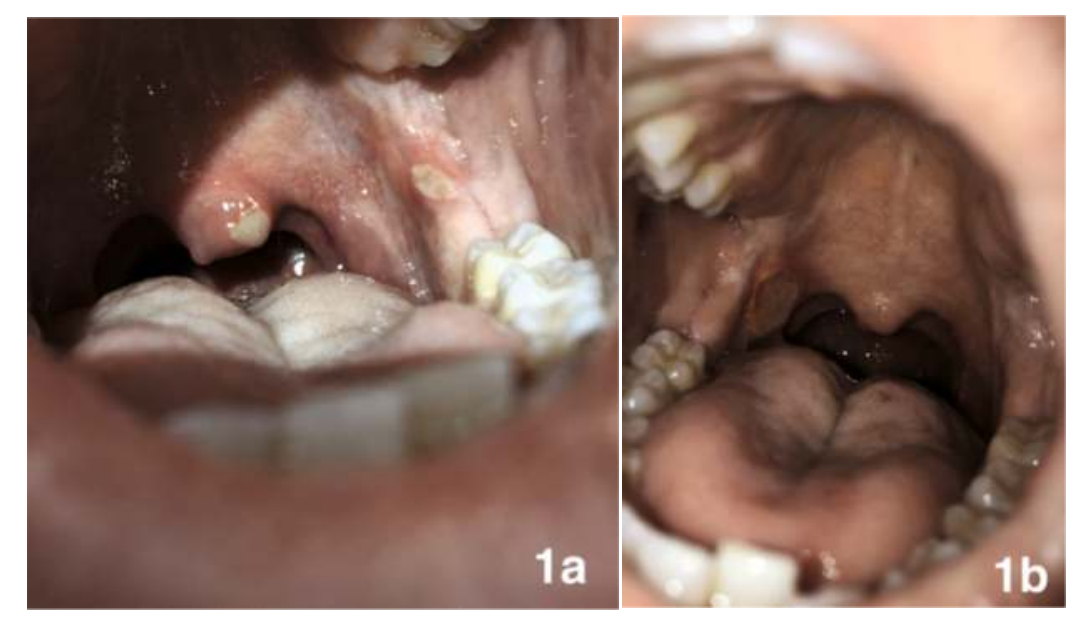

Figure $1-\mathbf{a}, \mathbf{b}-$ numerous painful ulcers of the oral cavity overlying the uvula, retromolar trigone and soft palate.

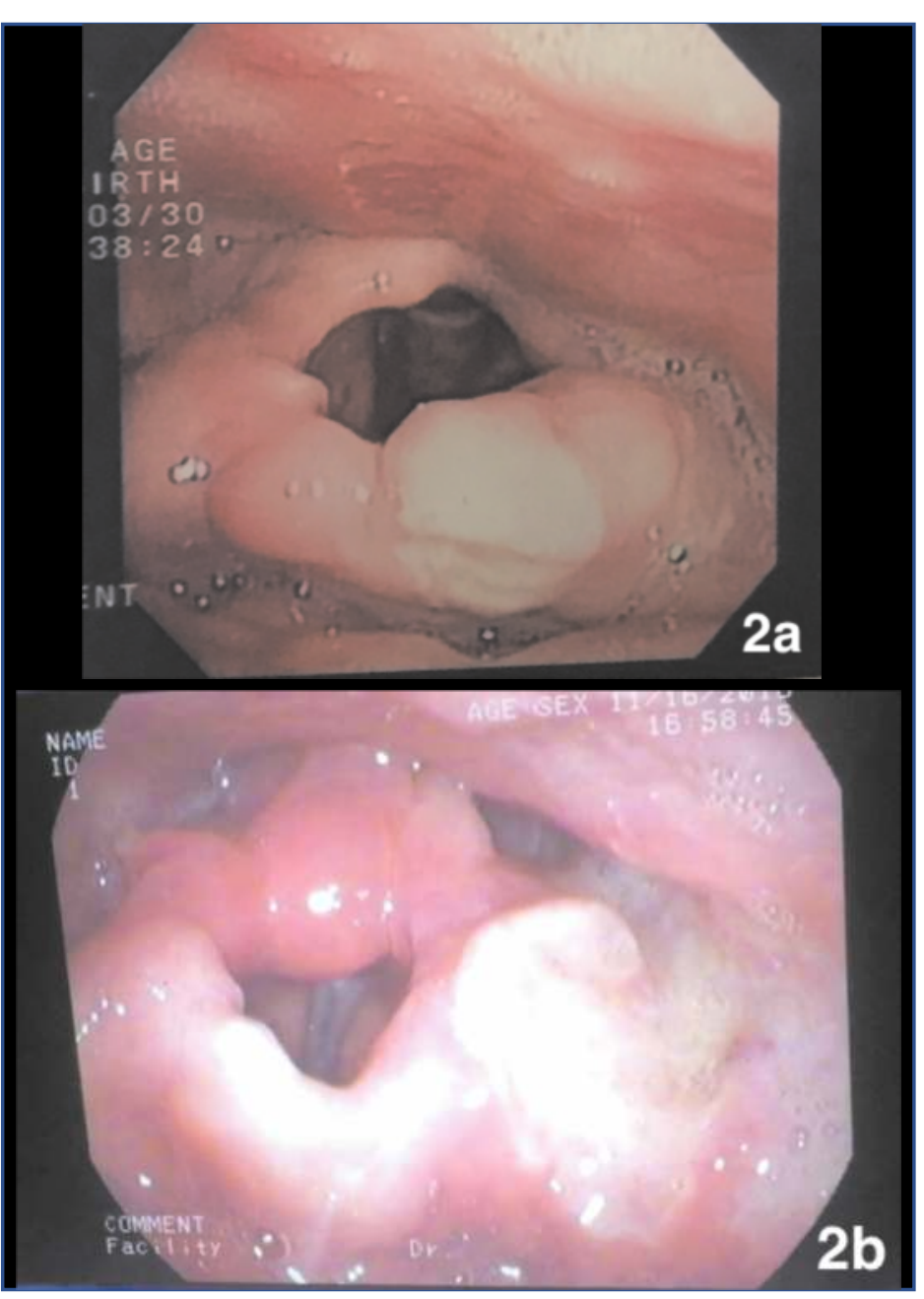

Figure 2 - Flexible laryngoscopy demonstrated general oedema and distortion of the supraglottis $\mathbf{a}-$ an initial ulcerative lesion on the epiglottis. b - Ulceration on the left aryepiglottic fold extending laterally into the left pyriform sinus with oedematous swelling of the arytenoids, but sparing of the vocal folds. Subsequent scarring of the epiglottis following healing of the initial epiglottic ulcer

\section{WHS}

Blackpool Teaching Hospitals NHS Foundation Trust

\section{DISCUSSION:}

- Diagnosing BD can be challenging and relies on the presence of appropriate clinical symptoms and signs given other possible causes are first excluded. Two international criteria can be used for guidance with diagnosis (Table 1, 2). BD is treated using the European League Against Rheumatism recommendations.

- This case illustrates the long journey often present from first symptoms to diagnosis and treatment (11 years) due to the staggered presentation of the disease.

- A systematic review in 2008 highlighted the ENT manifestations of BD. ${ }^{6}$ This included reports of patients developing laryngeal ulceration causing acute laryngeal inflammation and oedema, with risk of airway obstruction, and subsequent scarring.

- ENT manifestations in BD should be considered. Patients should be screened, with particular attention to the larynx, to allow for early recognition and treatment.

- In 2012, three national UK Behçet's Syndrome Centres of Excellence in London, Liverpool and Birmingham were established, providing an expert BD MDT.

- This case aims to raise awareness amongst the ENT community on these Centres of Excellence and their role in the diagnosis and treatment of such complex cases. 